\title{
Genotype Distribution of CNDP1 Polymorphisms in the Healthy Chinese Han Population: Association with HbAlc and Fasting Blood Glucose
}

\author{
Shiqi Zhang $\left(\mathbb{D}\right.$, ', Juan Xu $\mathbb{D}^{1},{ }^{1}$ Di Cui $\mathbb{D}^{1},{ }^{1}$ Shujuan Jiang $\mathbb{D}^{1}{ }^{1}$ Xin $X u \mathbb{D}^{1,2}$ Yi Zhang ${ }^{\mathbb{D}}{ }^{1}$ \\ Dongchun Zhu $\mathbb{D}^{1}{ }^{1}$ Li Xia $\mathbb{D}^{1},{ }^{1}$ Benito Yard $\mathbb{D},{ }^{2}$ Yonggui $W u\left(\mathbb{D},{ }^{3}\right.$ and Qiu Zhang $\mathbb{D}^{1}$ \\ ${ }^{1}$ Department of Endocrinology, The First Affiliated Hospital of Anhui Medical University, Hefei 230022, China \\ ${ }^{2}$ Vth Department of Medicine (Nephrology/Endocrinology/Rheumatology), University Medical Center Mannheim, \\ University of Heidelberg, Mannheim 68167, Germany \\ ${ }^{3}$ Department of Nephrology, The First Affiliated Hospital of Anhui Medical University, Hefei 230022, China
}

Correspondence should be addressed to Qiu Zhang; zhangqiu@ahmu.edu.cn

Received 16 January 2020; Revised 18 May 2020; Accepted 2 June 2020; Published 18 July 2020

Academic Editor: Janet H. Southerland

Copyright (C) 2020 Shiqi Zhang et al. This is an open access article distributed under the Creative Commons Attribution License, which permits unrestricted use, distribution, and reproduction in any medium, provided the original work is properly cited.

\begin{abstract}
We have previously reported that the CNDP1 (CTG) ${ }_{5}$ allele affords protection against diabetic nephropathy (DN) in patients with Type 2 diabetes (T2DM) of Caucasian origin. Because the incidence of ESRD attributable to both Type 1 diabetes (T1DM) and T2DM is higher among South Asian than Caucasian people, the present study assessed relevant CNDP1 polymorphisms and their association with metabolic parameters in the Chinese Han population. To this end, the $(\mathrm{CTG})_{\mathrm{n}}$ allele distribution along with 5 relevant SNPs in the CNDP1 gene, previously reported to be associated with DN in non (CTG) 5 carriers of AfroAmerican ethnicity, were determined in 663 healthy individuals. The (CTG) 6 homozygous genotype was the most prevalent $(84.5 \%)$ genotype in the Chinese Han population. The $(\mathrm{CTG})_{5}$ and $(\mathrm{CTG})_{4}$ alleles were present in a small minority of individuals accounting for $15.2 \%$ and $0.3 \%$ of genotypes with at least one (CTG) $)_{5}$ or one (CTG) $)_{4}$ allele, respectively. Only $0.5 \%$ of individuals carried the homozygous $(\mathrm{CTG})_{5}$ genotype and individuals carrying the homozygous (CTG) ${ }_{4}$ genotype were not found. The minor allele frequencies (MAFs) of the 5 SNP were 0.197 (C allele for rs4892247), 0.0855 (C allele for rs62099905), 0.085 ( $\mathrm{G}$ allele for rs62099906), 0.066 ( $\mathrm{T}$ allele for rs62099907), and 0.18 (A allele for rs72979715). All the SNPs except rs4892247 genotypes were in the Hardy-Weinberg equilibrium. Neither the (CTG) polymorphism nor the latter three SNPs reached significance when compared with different metabolic parameters. In contrast, individuals with the TT genotype of rs62099905 presented lower fasting blood glucose but higher HbA1c levels. In conclusion, the rs62099905 in the CNDP1 gene is associated with serum glucose levels in the healthy Chinese Han population, while for the CNDP1 (CTG) nolymorphism, no association with serological parameters was found.
\end{abstract}

\section{Introduction}

It has been reported that patients with Type 2 diabetes (T2DM) carrying the homozygous CNDP1 (CTG) $)_{5}$ genotype have a reduced risk to develop diabetic nephropathy (DN) as compared to T2DM patients carrying other genotypes [1]. The CNDP1 (CTG) ${ }_{\mathrm{n}}$ polymorphism is situated in the hydrophobic part of the carnosinase $1(\mathrm{CN}-1)$ signal peptide and may affect $\mathrm{CN}-1$ secretion into serum. In vitro studies have suggested that the shorter (CTG) $)_{5}$ allelic variant is less effi- ciently secreted [2], which might explain why (CTG) homozygous individuals have lower serum $\mathrm{CN}-1$ levels [1].

$\mathrm{CN}-1$ is a dipeptidase which selectively hydrolyzes the histidine-containing dipeptides (HCD) carnosine, anserine, and homocarnosine. These HCD have a broad spectrum of protective effects including antioxidative and antiglycative properties which may explain their beneficial effect in the context of diabetes and other disorders related to oxidative stress [3]. Indeed, oral carnosine supplementation ameliorates DN [4] and diabetic retinopathy (DR) [5] in Type 2 
and Type 1 diabetic models while overexpression of $\mathrm{CN}-1$ aggravates diabetes [4]. Although the number of studies performed in humans is limited, it seems that HCD also improves obesity and glycemic outcomes in men $[6,7]$. Yet, in humans, the protective effect of HCD is hampered by rapid hydrolysis of HCD because of high $\mathrm{CN}-1$ activities and concentrations in human serum. Hence, carnosine can only transiently be detected in human serum, shortly after oral ingestion in individuals with low CN-1 activity [8]. Based on the foregoing, it was hypothesized that the CNDP1 $(\mathrm{CTG})_{5}$ homozygous genotype affords protection because it is associated with lower serum $\mathrm{CN}-1$ activities which may facilitate higher tissue carnosine concentrations $[8,9]$.

The prevalence of the $(\mathrm{CTG})_{5}$ and $(\mathrm{CTG})_{6}$ alleles strongly varies among different ethnicities. The $(\mathrm{CTG})_{5}$ allele is relatively common in Caucasians with $38.6 \%$ and $29.3 \%$ (healthy controls vs. DN-ESRD patients) of individuals being homozygous for this allele [10]. The proportion is similar in India $(38.4 \%$ vs. $24.3 \%$, healthy controls vs. DN patients) [11]. However, in the East Asian population, the prevalence of the (CTG) $)_{5}$ allele seems to be much rarer. In the Netherlands, Surinamese migrants from South Asian origin have a significantly lower frequency of the homozygous $(\mathrm{CTG})_{5}$ genotype as compared to White Dutch $(23 \%$ vs. $41.3 \%$ ) [12]. In the Japanese population, frequencies of approximately $0.1 \%$ for this genotype are found in healthy individuals and in patients with DN [13]. Likewise, a study in peritoneal dialysis patients in Hong Kong revealed that the majority of patients $(80.3 \%)$ were homozygous for the $(\mathrm{CTG})_{6}$ allele, whereas the percentage of $(\mathrm{CTG})_{5}$ homozygous patients was less than $1 \%$ [14]. Hence, the frequency of the protective $(\mathrm{CTG})_{5}$ allele is considerably lower in the South Asian population, a population known to have a higher incidence of $\mathrm{DN}$ as compared to white European people $[15,16]$. Apart from the $(\mathrm{CTG})_{5}$ and $(\mathrm{CTG})_{6}$ alleles, also the $(\mathrm{CTG})_{4},(\mathrm{CTG})_{7}$, and $(\mathrm{CTG})_{8}$ alleles have been detected in different populations, albeit at significantly lower frequencies.

In addition to the (CTG) trinucleotide repeat polymorphism, single nucleotide polymorphisms (SNPs) within the CNDP1 gene have been postulated to affect renal complications in patients with T2DM. As such, the rs12604675 seems to be associated with overt proteinuria, but not with ESRD in Japanese women with T2DM [13]. Studies performed by McDonough pointed out that rs6566810, rs17089362 and rs6566810, rs17089362, rs890336 might mask the protection of the (CTG) $)_{5}$ allele [17].

Because current knowledge on the CNDP1 genotypes in the Chinese population is still fragmentary, this study was conducted to delineate the distribution of CNDP1 genotypes, with focus on relevant polymorphisms reported to affect disease course of-or susceptibility to $\mathrm{DN}$ and their potential association with biomarkers of renal function or other metabolic parameters in a nondiabetic Chinese population.

\section{Materials and Methods}

2.1. Subjects. A total of 813 subjects were recruited from the community clinics in the Xiyuan district in Hefei city (PR
China). Of these subjects, 150 were excluded because of incomplete genotyping data or missing informed consent. The remaining 663 subjects were all adults of Chinese Han origin and did not fulfill one or more of the following exclusion criteria: (1) cardiac disease; (2) brain disease; (3) hepatopathy; (4) kidney disease; (5) diabetes. All subjects gave their written informed consent before recruitment. This project is approved by the local ethical committee (No. PJ2018-05-09).

2.2. DNA Isolation. Genomic DNA was isolated from whole blood by the Genomic DNA extraction kit (Invitrogen, USA) according to manufacturer's instruction. DNA samples were stored at $-20^{\circ} \mathrm{C}$ until use.

2.3. CTG Polymorphism Genotyping. A 167 base pair fragment from exon 1 of the CNDP1 genes, which included the (CTG)n polymorphism, was amplified by standard PCR methods using a fluorescence-labeled forward primer $\left(5^{\prime}\right.$ FAM-AGGCAGCTGTGTGAGGTAAC-3') and an unlabeled reverse primer ( $5^{\prime}$-GGGTGAGGAGAACATGCC- $3^{\prime}$ ), respectively. Genotyping was performed according to fragment analysis on an ABI 3730XL (Applied Biosystems) sequencing platform.

2.4. SNPs Genotyping. Five SNPs within the CNDP1 gene, i.e., rs4892247, rs62099906, rs620999905, rs62099907, and rs72979715, were selected on the basis of previous publications $[13,14,17]$, provided that their minor allele frequency (MAF) in the studied Chinese Han population was more than 0.05. SNPs extension primers are shown in Table 1. All SNPs were genotyped by the SnaPshot kit (ABI) as previously described [18].

2.5. Statistics. Quantitative data are depicted as mean \pm standard deviation. Student $t$ test was used to compare differences between two groups, and One-way ANOVA was performed for more than two groups. Logarithm transformation was applied if data were not normally distributed. Correlations between categorical values were evaluated by the Pearson correlation coefficient. The continuous and categorical variables, which related to CNDP1 genotyping with a $p$ value of $<0.25$ of previous univariate analysis were selected for subsequent binary or multinomial regression analysis. Significance was defined according to a $p<0.05$. Deviations from Hardy-Weinberg equilibrium were evaluated with a Pearson's $\chi^{2}$ goodness-of-fit test. Statistical analyses were performed with SPSS16.0.

\section{Results}

Demographic data from the 663 included individuals are shown in Table 2. They ranged from 24 to 90 years of age, with mean values for waistline, body mass index (BMI), and blood pressure in the normal range. As expected from the inclusion criteria, there were no signs of diabetes or renal function impairment in this cohort of individuals. However, 92 out of 663 individuals were prediabetes (FBG (fasting blood glucose) 6.1-6.9 mmol/L or P2hBG (2 hours postprandial glucose) 7.8-11.0 mmol/L), 43 were obese (BMI more than $\left.28.5 \mathrm{~kg} / \mathrm{m}^{2}\right) ; 193$ individuals had hypertriglyceridemia 
TABLe 1: Primers for SNPs genotyping.

\begin{tabular}{|c|c|c|}
\hline & Primers & Primers for single-base extension reaction \\
\hline rs4892247-F & GTGAAGGGAAAATCACAACACTTGC & TA АTATACTCTTCCАTCAАCA^АCTCATTA \\
\hline rs4892247-R & TGCTGCGAGATACTGGGTG & 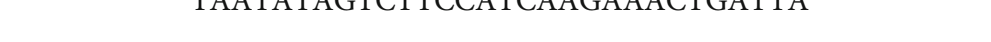 \\
\hline rs62099905-F & CACTCTGTGATCCTCCCACC & TAGGTATGCACCACCTGGCCTGGCTAAGTTTTAAAATCTGATTTCG \\
\hline rs62099905-R & AAGATGCCCATTCCAGGTC & CTTA \\
\hline rs62099906-F & GCCCAGGAATCAGAGCAGA & GCACTTCT А \\
\hline rs62099906-R & ССTCTTTCTTTGGCCTCCC & 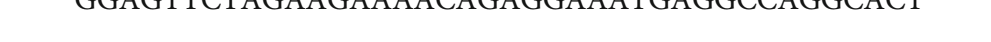 \\
\hline rs62099907-F & GCCCAGGAATCAGAGCAGA & AATGAGGCCAGGCACTATGGCTCACACCTGTAATACTAGCATTTTG \\
\hline rs62099907-R & ATTTTTTTTAGAGATGGGGAC & GGAGGCCAAAGAA \\
\hline rs72979715-F & CACATGGGCATAGGATGGA & CACACATACACAGGGAGAACATCATAAGAAGATGAGGGCAGAGATC \\
\hline rs72979715-R & СССTACACTGGGTGGCTTA & GGGTGATGCTTGTACAAGACAA G \\
\hline
\end{tabular}

TABle 2: Clinical and demographic data of all subjects.

\begin{tabular}{lcc}
\hline & Female $(n=311)$ & Male $(n=352)$ \\
\hline Age (year) & $55.44 \pm 14.39$ & $52.39 \pm 12.77$ \\
Waistline $(\mathrm{cm})$ & $78.75 \pm 15.03$ & $79.73 \pm 22.24$ \\
Body mass index $\left(\mathrm{kg} / \mathrm{m}^{2}\right)$ & $23.21 \pm 3.59$ & $24.58 \pm 4.13$ \\
Systolic blood pressure $(\mathrm{mmHg})$ & $121.28 \pm 20.25$ & $126.34 \pm 18.57$ \\
Diastolic blood pressure $(\mathrm{mmHg})$ & $76.61 \pm 11.71$ & $82.39 \pm 10.87$ \\
Triglyceride $(\mathrm{mmol} / \mathrm{L})$ & $1.53 \pm 1.12$ & $1.71 \pm 1.30$ \\
Total cholesterol $(\mathrm{mmol} / \mathrm{L})$ & $4.86 \pm 0.93$ & $4.81 \pm 3.65$ \\
Serum urea nitrogen $(\mathrm{mmol} / \mathrm{L})$ & $4.62 \pm 1.20$ & $4.75 \pm 1.20$ \\
Creatinine $(\mu \mathrm{mol} / \mathrm{L})$ & $75.05 \pm 11.27$ & $74.86 \pm 12.22$ \\
eGFR $(\mathrm{MDRD})(\mathrm{mL} / \mathrm{min})$ & $85.75 \pm 19.31$ & $106.24 \pm 28.38$ \\
Urinary ACR $(\mathrm{mg} / \mathrm{g})$ & $1.74 \pm 2.85$ & $1.97 \pm 5.20$ \\
Skin AGEs & $70.58 \pm 11.15$ & $70.80 \pm 9.75$ \\
HbAlc $\%)$ & $5.75 \pm 0.56$ & $5.76 \pm 0.85$ \\
Fasting plasma glucose $(\mathrm{mmol} / \mathrm{L})$ & $5.19 \pm 0.85$ & $5.41 \pm 1.10$ \\
2 hours postprandial blood glucose $(\mathrm{mmol} / \mathrm{L})$ & $6.94 \pm 2.84$ & $7.04 \pm 2.81$ \\
Fasting C peptide $(\mathrm{ng} / \mathrm{mL})$ & $1.02 \pm 1.28$ & $0.93 \pm 0.70$ \\
\hline
\end{tabular}

HbAlc: Hemoglobin $\beta$ chain (blood) -N- (1-deoxyfructose-1-yl) hemoglobin $\beta$ chain.

(serum triglyceride more than $1.7 \mathrm{mmol} / \mathrm{L}$ ); and 60 individuals had hypercholesterolemia.

Genotyping data for the CNDP1 $(\mathrm{CTG})_{\mathrm{n}}$ genotype distribution was complete for all 663 individuals as depicted in Table 3. The majority of individuals $(84.5 \%)$ carried the homozygous $(\mathrm{CTG})_{6}$ genotype, followed by individuals that were heterozygous for the $(\mathrm{CTG})_{5}$ and $(\mathrm{CTG})_{6}$ allele (14.8\%). Only 3 out of 663 individuals $(0.5 \%)$ were homozygous for the $(\mathrm{CTG})_{5}$ allele, and likewise, the $(\mathrm{CTG})_{4}$ allele was found in a small minority of individuals ( 2 out of 663 , $0.3 \%)$ and only in combination with the (CTG) ${ }_{6}$ allele.

Genotyping data for the 5 selected SNPs were complete in 642 individuals for rs4892247; 641 for rs62099905; 644 for rs62099906, rs62099907, and rs72979715. SNPs were selected on the basis of previous publications [13, 14, 17], showing an association with renal parameters in patients with T2DM, provided that the minor allele frequency
TABLE 3: Distribution of CNDP1 genotyping in China.

\begin{tabular}{lcc}
\hline CNDP1 genotyping & $\mathrm{n}$ & Percentage (\%) \\
\hline${\text { (CTG })_{4-6}}_{(\mathrm{CTG})_{5-5}}$ & 2 & 0.3 \\
$(\mathrm{CTG})_{5-6}$ & 3 & 0.5 \\
$(\mathrm{CTG})_{6-6}$ & 98 & 14.8 \\
Total & 559 & 84.5 \\
\hline
\end{tabular}

(MAF) of the selected SNPs was more than 0.05 for the Chinese Han population in the 1000 Genomes database. The frequencies for the different loci are shown in detail in Table 4. The minor alleles and their calculated MAF as well as the MAF in the 1000 genomes database are shown in Table 4. Moreover, all SNPs except rs4892247 were in Hardy-Weinberg equilibrium $(p>0.05)$. 
TABLE 4: Minor allele frequencies and genotype distribution of CNDP1 SNPs.

\begin{tabular}{|c|c|c|c|c|c|c|}
\hline & Minor allele & MAF & MAF in 1000 genomes & SNP & $n$ & Valid percent (\%) \\
\hline \multirow{3}{*}{ Rs4892247 genotyped $n=642$} & $\mathrm{C}$ & 0.197 & 0.2787 & $\mathrm{CC}$ & 4 & 0.6 \\
\hline & & & & TT & 393 & 61.2 \\
\hline & & & & $\mathrm{TC}$ & 245 & 38.2 \\
\hline \multirow{3}{*}{ Rs62099906 genotyped $n=644$} & G & 0.085 & 0.1787 & AA & 538 & 83.5 \\
\hline & & & & AG & 102 & 15.8 \\
\hline & & & & GG & 4 & 0.6 \\
\hline \multirow{3}{*}{ Rs62099905 genotyped $n=641$} & $\mathrm{C}$ & 0.0855 & 0.1755 & $\mathrm{CC}$ & 4 & 0.6 \\
\hline & & & & $\mathrm{CT}$ & 102 & 15.9 \\
\hline & & & & TT & 535 & 83.5 \\
\hline \multirow{2}{*}{ Rs62099907 genotyped $n=644$} & $\mathrm{~T}$ & 0.066 & 0.1755 & AA & 559 & 86.8 \\
\hline & & & & AT & 85 & 13.2 \\
\hline \multirow{3}{*}{ Rs72979715 genotyped $n=644$} & A & 0.18 & 0.1154 & AA & 20 & 3.1 \\
\hline & & & & AG & 192 & 29.8 \\
\hline & & & & GG & 432 & 67.1 \\
\hline
\end{tabular}

TABLE 5: Summary of logistic regression test of variables in rs62099905 genotypes.

\begin{tabular}{|c|c|c|c|c|c|c|}
\hline \multirow[b]{2}{*}{ Variables } & \multicolumn{3}{|c|}{ Univariate analysis } & \multicolumn{3}{|c|}{ Binary logistic regression analysis } \\
\hline & $\mathrm{CT}$ & TT & $p$ value & $B$ & OR $(95 \% \mathrm{CI})$ & $p$ value \\
\hline$N(\%)$ & $102(15.9)$ & $535(83.5)$ & - & - & - & - \\
\hline sBP (mmHg) & $127.90 \pm 20.65$ & $123.45 \pm 19.37$ & $0.055 *$ & -0.01 & $0.990(0.978-1.002)$ & 0.096 \\
\hline Serum urea nitrogen $(\mathrm{mmol} / \mathrm{L})$ & $4.81 \pm 1.37$ & $4.65 \pm 1.16$ & $0.249 *$ & -0.087 & $0.917(0.760-1.106)$ & 0.365 \\
\hline HbAlc $(\%)$ & $5.68 \pm 0.58$ & $5.77 \pm 0.76$ & $0.186 *$ & 0.465 & $1.592(1.028-2.465)$ & $0.037 * *$ \\
\hline $\mathrm{FBG}(\mathrm{mmol} / \mathrm{L})$ & $5.48 \pm 1.13$ & $5.27 \pm 0.97$ & $0.073 *$ & -0.276 & $0.759(0.577-0.998)$ & $0.048 * *$ \\
\hline Fasting C-peptide (ng/mL) & $0.86 \pm 0.62$ & $0.98 \pm 1.08$ & $0.117 *$ & 0.229 & $1.258(0.886-1.784)$ & 0.199 \\
\hline
\end{tabular}

${ }^{*} p<0.25 ;{ }^{* *} p<0.05$. B: regression coefficient. —: not applicable. sBP: systolic blood pressure. HbA1c: Hemoglobin $\beta$ chain (blood) -N- (1-deoxyfructose-1-yl) hemoglobin $\beta$ chain. FBG: fasting blood glucose.

We next assessed if the (CTG) $)_{n}$ polymorphism or the rest four SNPs showed an association with renal function or metabolic parameters. For the former polymorphism, patients were stratified on the basis of being homozygous for the $(\mathrm{CTG})_{6}$ allele $(n=559)$ vs. all other genotypes $(n=104)$. Since urinary ACR was not normally distributed in the dataset, a log transformation was performed for ACR to reach normal distribution (shown as Log ACR). In independent $t$ test analysis, only 3 variables showed a $p$ value less than 0.25 , including systolic blood pressure (sBP), diastolic blood pressure (dBP), and skin AGEs (data not shown). These 3 variables were subsequently included in the binary logistic regression analysis but showed no significant association with the (CTG) nolymorphism (data not shown).

For associations with the selected SNP the homozygous genotypes GG in rs62099906 $(n=4), \mathrm{CC}$ in rs62099905 $(n=4)$, and AA allele in rs72979715 $(n=20)$ were excluded from the analysis due to the small sample size. Hence, independent $t$ tests for the association were later performed between AA and AG genotypes in rs62099906, between CT and TT genotypes in rs62099905, between AA and AT genotypes in rs62099907, and between AG and GG genotypes in rs72979715. Variables with $p<0.25$ were subsequently selected for binary logistic analysis.
Although in univariate analysis, a number of parameters reached a threshold $p$ values $<0.25$, in the multivariate analysis, no significant associations for rs62099906, rs62099907, and rs72979715 were found. In contrast, rs62099905 (Table 5) showed significant associations in the multivariate model. Rs62099905 was associated HbA1c and FBG. Individuals with the TT genotype of rs62099905 displayed higher HbA1c but lower FBG levels (Figure 1, CC vs. CT vs. TT: $\mathrm{HbAlc}(\%): 5.63 \pm 0.35$ vs. $5.68 \pm 0.58$ vs. $5.77 \pm 0.76$; FBG $(\mathrm{mmol} / \mathrm{L}): 5.48 \pm 0.61$ vs. $5.48 \pm 1.13$ vs. $5.27 \pm 0.97)$. The P2hBG levels between CT and TT allele were however not significant although the levels were slightly higher in the TT allele (CC vs. CT vs. TT: P2hBG (mmol/L): $6.77 \pm 1.34$ vs. $6.92 \pm 2.87$ vs. $7.01 \pm 2.85)$.

\section{Discussion}

CN-1 gained scientific prominence by the finding that polymorphisms in the CNDP1 gene may affect chronic kidney disease in diabetic and nondiabetic patients [1]. A (CTG) trinucleotide repeat polymorphism in the signal peptide of $\mathrm{CN}-1$ has mostly been studied in this context and reported to affect $\mathrm{CN}-1$ secretion [2]. The $(\mathrm{CTG})_{5}$ allele seems to afford protection in patients with $\mathrm{T} 2 \mathrm{DM}$ as the frequency 


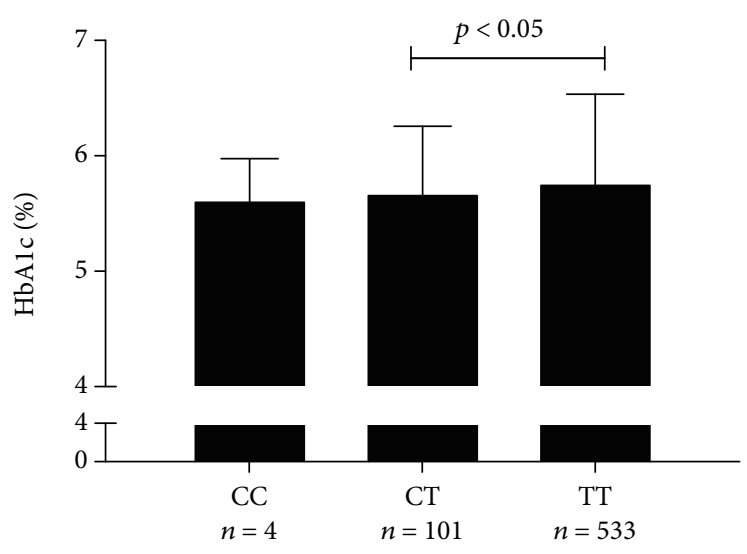

(a)

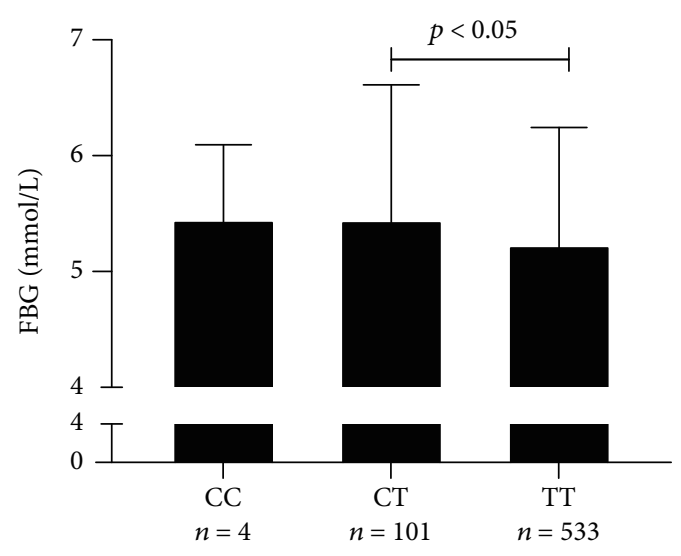

(b)

FIGURE 1: rs62099905 polymorphism in CNDP1 gene correlated with HbA1c and fasting blood glucose levels. Legend: TT homozygosity in rs62099905 showed higher HbA1c levels (a) but lower FBG levels (b) as compared to CT heterozygosity. CC homozygosity which was not included in this statistical analysis had almost similar HbA1c and FBG levels as compared to CT heterozygosity. Abbreviations: HbA1c: Hemoglobin $\beta$ chain (blood) -N- (1-deoxyfructose-1-yl) hemoglobin $\beta$ chain; FBG: fasting blood glucose.

of the homozygous (CTG) ${ }_{5}$ genotype is significantly lower in patients with DN [1]. Since the South Asian population is much more prone to develop DN than the Caucasian population $[15,16,19]$, the intention of the current study was to assess the genotype distribution of polymorphisms within the CNDP1 gene, previously reported to affect disease course of-or susceptibility to DN. We studied a healthy population to avoid a selection bias due to the high all-cause mortality in diabetic patients with impaired renal function. Notwithstanding this, we analyzed ACR and eGFR to assess for potential associations between these biomarkers and certain genotype.

The prevalence of the different CNDP1 (CTG) $)_{n}$ alleles varies among different ethnicities. While the $(\mathrm{CTG})_{5}$ and $(\mathrm{CTG})_{6}$ alleles are common in the Caucasian population, the former allele is not frequently found in the Asian population $[12,14]$. Our study is in line with this notion as only a remarkably low proportion (0.5\%) of our studied Chinese Han population was homozygous for the $(\mathrm{CTG})_{5}$ allele. This contrasted the relatively high proportion $(84.5 \%)$ of individuals homozygous for the (CTG) ${ }_{6}$ allele, a finding that was also reported for peritoneal dialysis patients in Hong Kong [14]. Our data are also similar to that reported in the Japanese population [13]. It was found that the homozygous $(\mathrm{CTG})_{6}$ genotype was most common amongst subjects followed by subjects heterozygous for the $(\mathrm{CTG})_{6}$ and $(\mathrm{CTG})_{5}$ allele [13]. The Hong Kong, the Japanese, and our study all three report on lower frequencies of the homozygous $(\mathrm{CTG})_{5}$ as compared to the study of Mooyaart et al., in which Surinamese migrants from South Asian origin were included [12].

Neither ACR nor eGFR showed a significant relation with the CNDP1 (CTG) genotypes in multivariant analysis. This finding is not unexpected because in a healthy population, ACR and eGFR are in a normal range, and to our knowledge, there is no direct evidence that links carnosine or $\mathrm{CN}-1$ to these parameters in healthy individuals. Moreover, ACR and eGFR values were mostly below $5 \mathrm{mg} / \mathrm{g}$, respectively, more than $60 \mathrm{~mL} / \mathrm{min}$ with only small deviation as compared to diabetic patients.

The MAFs of the 5 SNPs we analyzed within the CNDP1 gene are roughly consistent with published data, albeit that we observed a slightly lower MAF in rs4892247, rs62099906, rs62099905, and in rs62099907 and a slightly higher MAF in rs72979715. McDonough et al. reported that rs4892247 located in intron 9 of the CNDP1 gene is associated with end-stage renal disease (ESRD) caused by diabetes [17]. Likewise, an association between chronic kidney disease and rs4892247 has been reported [20]. Unfortunately, the distribution of rs 4892247 was not in Hardy-Weinberg equilibrium in our study. A larger cohort is needed to reach genetic equilibrium. For the rs62099905, significant associations were found, i.e., individuals with the TT genotype of rs62099905 presented with lower fasting blood glucose but higher HbAlc levels. This can be explained by the finding that $\mathrm{P} 2 \mathrm{hBG}$ values contribute more to $\mathrm{HbAlc}$ in the Chinese population [21] and by the finding that individuals with the rs62099905 TT genotype displayed slightly higher P2hBG values. The single fasting or postprandial glucose level testing in our study, which might vary due to different factors, was also not a good predictor for HbAlc. Whether individuals carrying the TT genotypes of rs4892247 and/or rs62099905 display higher serum $\mathrm{CN}-1$ activities or concentrations is currently being studied.

Although the intention of our study was to describe the genotype distribution of relevant polymorphisms within the CNDP1 gene in the Chinese Han population, one limitation of this study is the lack of translating the individual genotypes to $\mathrm{CN}-1$ concentrations or activities. In keeping with the notion that apart from the $(\mathrm{CTG})_{\mathrm{n}}$ polymorphism other factors, e.g., age and gender, are known to affect serum $\mathrm{CN}$ 1 concentration [22, 23], the large proportion of individuals carrying the homozygous (CTG) 6 genotype offers the opportunity to test the influence of the other CNDP1 polymorphisms on $\mathrm{CN}-1$ concentrations. These studies are currently conducted. Further studies are also required to 
assess if and how serum triglycerides and $\mathrm{HbAlc}$ are affected by carnosine and if this correlates with CNDP1 genotypes that are associated with high or low serum CN-1 concentrations.

In conclusion, our data confirm and extent previous studies on genotype distribution of DN relevant polymorphisms within the CNDP1 gene. We also for the first-time report on an association between fasting blood glucose and HbA1c and the SNP within the CNDP1 gene. Further epidemiological studies are warranted to confirm these findings, preferably also in different ethnicities.

\section{Data Availability}

The genotyping data used to support the findings of this study are restricted by the local ethics committee in order to protect patient privacy. Data are available from Dr. Shiqi Zhang (zhangshiqi@ahmu.edu.cn), the first author of this publication, for researchers who meet the criteria for access to confidential data.

\section{Conflicts of Interest}

The authors declare that there is no conflict of interest associated with this manuscript.

\section{Authors' Contributions}

Yonggui $\mathrm{Wu}$ and Qiu Zhang contributed equally to this work.

\section{Acknowledgments}

The authors would like to thank all involved in Xiyuan district for supporting the recruitment of patients, and Yang Zhang, Yikun Wang (Department of Bio-Medical Optics Research Lab, Hefei Institutes of Physical Science, Chinese Academy of Sciences, Hefei 230031, China) for their assistance in providing skin Auto-fluorescence Reader. This work was supported by the Sino-German Center for Research Promotion (SGC) and National Natural Science Foundation of China (NSFC) to project "The role of carnosinase-1 and its mechanism in the progression of chronic kidney disease (No. 81761138042)" and Young Scientists Cultivation program in The first affiliated hospital of Anhui Medical University to project "The role of carnosinase-1 in diabetic nephropathy and other diabetic complications (2017kj16)".

\section{References}

[1] B. Janssen, D. Hohenadel, P. Brinkkoetter et al., "Carnosine as a protective factor in diabetic nephropathy: association with a leucine repeat of the carnosinase gene CNDP1," Diabetes, vol. 54, no. 8, pp. 2320-2327, 2005.

[2] E. Riedl, H. Koeppel, P. Brinkkoetter et al., "A CTG polymorphism in the CNDP1 gene determines the secretion of serum carnosinase in Cos-7 transfected cells," Diabetes, vol. 56, no. 9, pp. 2410-2413, 2007.
[3] A. A. Boldyrev, G. Aldini, and W. Derave, "Physiology and pathophysiology of carnosine," Physiological Reviews, vol. 93, no. 4, pp. 1803-1845, 2013.

[4] S. Sauerhöfer, G. Yuan, G. S. Braun et al., "L-carnosine, a substrate of carnosinase-1, influences glucose metabolism," Diabetes, vol. 56, no. 10, pp. 2425-2432, 2007.

[5] F. Pfister, E. Riedl, Q. Wang et al., "Oral carnosine supplementation prevents vascular damage in experimental diabetic retinopathy," Cellular Physiology and Biochemistry, vol. 28, no. 1, pp. 125-136, 2011.

[6] K. Menon, C. Marquina, D. Liew, A. Mousa, and B. de Courten, "Histidine-containing dipeptides reduce central obesity and improve glycaemic outcomes: a systematic review and meta-analysis of randomized controlled trials," Obesity Reviews, vol. 21, no. 3, article e12975, 2020.

[7] S. Karkabounas, N. Papadopoulos, C. Anastasiadou et al., "Effects of $\alpha$-lipoic acid, carnosine, and thiamine supplementation in obese patients with type 2 diabetes mellitus: a randomized, Double-Blind Study," Journal of Medicinal Food, vol. 21, no. 12, pp. 1197-1203, 2018.

[8] I. Everaert, Y. Taes, E. de Heer et al., "Low plasma carnosinase activity promotes carnosinemia after carnosine ingestion in humans," American Journal of Physiology-Renal Physiology, vol. 302, no. 12, pp. F1537-F1544, 2012.

[9] I. Everaert, J. He, M. Hanssens et al., "Carnosinase-1 overexpression, but not aerobic exercise training, affects the development of diabetic nephropathy in BTBRob/obmice," American Journal of Physiology-Renal Physiology, vol. 318, no. 4, pp. F1030-F1040, 2020.

[10] B. I. Freedman, P. J. Hicks, M. M. Sale et al., "A leucine repeat in the carnosinase gene CNDP1 is associated with diabetic endstage renal disease in European Americans," Nephrology, Dialysis, Transplantation, vol. 22, no. 4, pp. 1131-1135, 2007.

[11] A. K. Yadav, N. Sinha, V. Kumar, A. Bhansali, P. Dutta, and V. Jha, "Association of CTG repeat polymorphism in carnosine dipeptidase 1 (CNDP1) gene with diabetic nephropathy in north Indians," The Indian Journal of Medical Research, vol. 144, no. 1, pp. 32-37, 2016.

[12] A. L. Mooyaart, I. G. M. van Valkengoed, P. K. C. Shaw et al., "Lower frequency of the 5/5 homozygous CNDP1 genotype in South Asian Surinamese," Diabetes Research and Clinical Practice, vol. 85, no. 3, pp. 272-278, 2009.

[13] M. Kurashige, M. Imamura, S. I. Araki et al., "The influence of a single nucleotide polymorphism within CNDP1 on susceptibility to diabetic nephropathy in Japanese women with type 2 diabetes," PLoS One, vol. 8, no. 1, article e54064, 2013.

[14] P. Y. K. Poon, C. C. Szeto, B. C. H. Kwan, K. M. Chow, and P. K. T. Li, "Relationship between carnosinase gene CNDP1 leucine repeat polymorphism and the clinical outcome of Chinese PD patients," Clinical Nephrology, vol. 74, no. 11, pp. 343-345, 2010.

[15] A. C. Burden, P. C. McNally, J. Feehally, and J. Walls, "Increased incidence of end-stage renal failure secondary to diabetes mellitus in Asian ethnic groups in the United Kingdom," Diabetic Medicine, vol. 9, no. 7, pp. 641-645, 1992.

[16] A. A. Lopes, "End-stage renal disease due to diabetes in racia1/ethnic minorities and disadvantaged populations," Ethnicity \& Disease, vol. 19, 1 Supplement 1, pp. S1-47-S1-51, 2009.

[17] C. W. McDonough, P. J. Hicks, L. Lu, C. D. Langefeld, B. I. Freedman, and D. W. Bowden, "The influence of carnosinase gene polymorphisms on diabetic nephropathy risk in 
African-Americans," Human Genetics, vol. 126, no. 2, pp. 265275, 2009.

[18] M. Bujalkova, K. Zavodna, T. Krivulcik et al., "Multiplex SNaPshot genotyping for detecting loss of heterozygosity in the mismatch-repair genes MLH1 and $M S H 2$ in microsatellite-unstable tumors," Clinical Chemistry, vol. 54, no. 11, pp. 1844-1854, 2008.

[19] P. K. Chandie Shaw, F. Baboe, L. A. van Es et al., "South-Asian type 2 diabetic patients have higher incidence and faster progression of renal disease compared with Dutch-European diabetic patients," Diabetes Care, vol. 29, no. 6, pp. 1383-1385, 2006.

[20] J. Guo, L. M. Chen, B. C. Chang, M. Y. Zheng, and J. J. Wen, "Association analysis of polymorphism in CNDP1 gene and chronic kidney disease in type 2 diabetic Chinese Han population," Chinese Journal of Diabetes, vol. 24, no. 1, pp. 23-27, 2016.

[21] R. Yan, Y. Hu, F. Li et al., "Contributions of fasting and postprandial glucose concentrations to haemoglobin Alc in drug-naïve mal-glucose metabolism in Chinese population using continuous glucose monitoring system," International Journal of Endocrinology, vol. 2019, Article ID 1267475, 5 pages, 2019.

[22] A. L. Mooyaart, A. Zutinic, S. J. L. Bakker et al., "Association between CNDP1 genotype and diabetic nephropathy is sex specific," Diabetes, vol. 59, no. 6, pp. 1555-1559, 2010.

[23] J. F. Lenney, R. P. George, A. M. Weiss, C. M. Kucera, P. W. H. Chan, and G. S. Rinzler, "Human serum carnosinase: characterization, distinction from cellular carnosinase, and activation by cadmium," Clinica Chimica Acta, vol. 123, no. 3, pp. 221$231,1982$. 